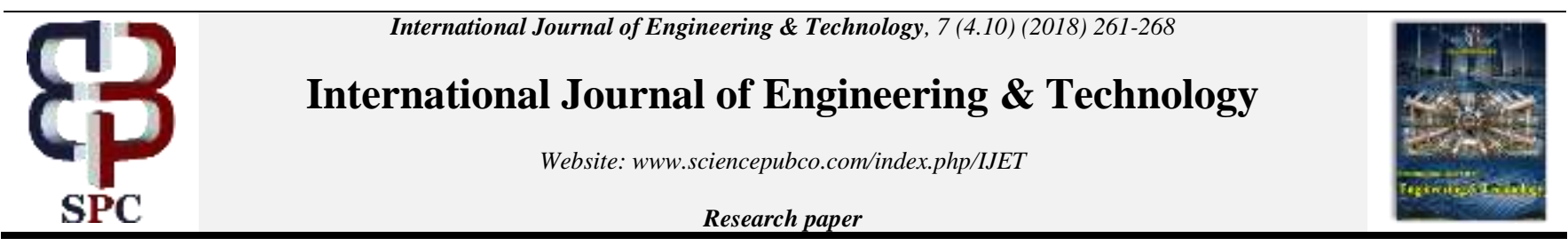

\title{
Effect of Thermal Radiation on Heat Transfer of Ferrofluid Over a Stretching Cylinder with Convective Heating
}

\author{
K. Gangadhar ${ }^{1}$, K.Keziya ${ }^{2}$ and B. Rushi Kumar ${ }^{3 *}$ \\ ${ }^{1,2}$ Department of Mathematics, Acharya Nagarjuna University, Ongole, Andhra Pradesh-523001,India \\ ${ }^{3}$ Department of Mathematics, School of Advanced Sciences, VIT, Vellore-632014,TN, India \\ *Corresponding author E-mail: rushikumar@vit.ac.in
}

\begin{abstract}
In view of this we scrutinize the numerical solution using the Kellor box method for the natural differential equations which describes the MHD flow of ferrofluid over a stretching cylinder with thermal radiation and convective heating. Water as convectional base fluid containing nano particles of magnetite $\left(\mathrm{Fe}_{3} \mathrm{O}_{4}\right)$ is taken up. Comparison between magnetite $\left(\mathrm{Fe}_{3} \mathrm{O}_{4}\right)$ and non-magnatic $\left(\mathrm{Al}_{2} \mathrm{O}_{3}\right)$ nanoparticles is also made. The relevant physical parameters appearing in velocity and temperature distributions are analyzed and examined with the help of Fig.s. To examine the correctness of the method an anology has been made with some earlier published results. It is noticed that by increase the strength of magnetic field, the percent difference in the heat transfer rate of magnetic nano particles with $\mathrm{Al}_{2} \mathrm{O}_{3}$ decrease. Further, convective heating and thermal radiation are highly influenced the temperature distribution of the ferrofluid.
\end{abstract}

Keywords: Ferrofluid, stretching cylinder, MHD, convective heating, thermal radiation.

\section{Introduction}

The detailed examination of Nanofluids has been subjected of covering and exploring the aftereffect or consequences of rising thermal conductivity heat transfer procedure .Nanofluid is propose to refer a fluid in that nanometer-magnitude molecules are suspended in classical heat transfer absolutely necessary fluids $[1,2]$. The fluids which are considered as bad transfer of heat are Water, encom-passing oil, and ethylene glycol. In various engineering applications like microelectronics, heat interchangers, refrigerating of electronic apparatus Nanofluids are used. A guanine studies of this wonderful topic together with the theoretical as well as experimental data can be much benefited in the literature [3-11]. More precisely In non-conducting liquids such as heptanes, water, kerosene, and hydrocarbons, the magnetic Nanofluids (Ferro fluids) those are colloidal suspension of magnetic nano particles like cobalt, ferrite, magnetite are dispersed. Ferrofluids are quite useful to explain different engineering applications like drug targeting technology, mega-phones, rotary shaft seals and bumpers. In the literatures [4-15] we can found the dissimilar applications of ferrofluids if we observe it in detailed. The impact of nanoparticle migration on thermal performance of ferrofluid flow inside a vertical micro ring examine by Malvandi et al [16], a few days ago. Heysiattalab et al [17] deeply examine the anisotropic approach of ferrofluids at film wise amplification on a vertical plate with a changeable directional magnetic field. Nanoparticle migration effects, and thee condensate falling film of ferrofluids in the presence of anisotropic behavior of thermal conductivity is analysed theoretically by Malvandi et al [18]. Rashad [19] was observed that the consequence of partial slip over a MHD flow of mixed convection of ferrofluid on a radiating wedge of non-isothermal. In another study, In the presence of slip effect how the boundary layer of a ferro fluid flows on semi infinite inclined plate also studied by Rashad [20].
The processes having high temperature like nuclear reactors, combustion turbines and storage of thermal energy etc... the analysis of heat transfer by convection accompanied by thermal radiation has more significance. In the work [21] the authors were explored the thermal radiation effects based on Rooseland diffusion approximation over a combined convection across vertical plate with free stream of uniform surface temperature and uniform velocity. The thermal radiation of gray fluid that emits as well as absorbs radiation in a non-scattering medium was examined in [22]. Authors in [23] were examined the radiative flow in the existence of magnetic field. The authors in [24] were explained the impact of thermal radiation on the boundary layer flow by an exponentially stretching sheet. The authors of [25] examined the impact of thermal radiation on MHD flow of a second grade fluid. In [26] the authors were assessed that the consequences of radiation over natural streamline flow from a horizontal circular cylinder. Finally it was concluded that increase in radiation results increase in velocity as well as thermal boundary layer thickness. Ahmad et al. [27] examined the influence of thermal radiation over mixed convection boundary layer flow of a visco elastic fluid over a circular cylinder with constant surface temperature.

By the work of Aziz [28], on the boundary layer flow with convective surface boundary condition have acquired more attentiveness, in recent years. In this work he also explored by connective surface boundary condition the thermal boundary layer flow over a flat plate. Afterwards Makinde and Aziz [29] together by taking the same connective boundary conditions they explained the boundary layer flow of a nanofluid past over a stretching sheet. Shehzad et al. [30] by using convective surface boundary conditions explained exact solution of three-dimensional flow of Jeffery fluid. Hayat et al.[31], by using convective surface boundary conditions he scrutinized the three dimensional boundary layer flow of Maxwell fluid on stretching surface. Zaih et al. [32] studied the effect of thermal radiation and convective boundary conditions on William son fluid over an exponentially stretching sheet. 
So far no studies have been made to scrutinize the out-turn of thermal radiation on MHD boundary layer flow of a ferrofluid on stretching cylinder with convective heating. The Keller box method used gives us a numerical solution of the equation obtained. This only describes the problem, after similarity functions. The effects of the embedded flow controlling parameters on various issues like fluid velocity, temperature skin friction and heat transfer rate revealed in the similarity solution presented in order to predict heat transfer characteristics. A comparative study is also presented below.

\section{Mathematical Analysis}

Take a ferrofluid, In that we introduce a cylinder. Suppose the radius of cylinder is ' $a$ ', here it is assumed that the cylinder is stretching along its axis. The velocity of the fluid, $U_{w}=U_{0}$, the heat flux on the cylinder surface is $q_{w}=T_{0}$, and $U_{0}, T_{0}$ are the functions of $\frac{x}{l}$.A boundary layer is formed on the circular cylinder. Suppose the uniform magnetic field of intensity $B_{0}$ acts along the radial direction due to this assumption for small magnetic Reynolds number the impact of induced magnetic field is almost negligible. Now the above system is explained by the following equations

$$
\begin{aligned}
& \frac{\partial}{\partial x}(r u)+\frac{\partial}{\partial r}(r v)=0 \\
& \mathrm{u} \frac{\partial \mathrm{u}}{\partial \mathrm{x}}+\mathrm{v} \frac{\partial \mathrm{u}}{\partial \mathrm{r}}=\frac{\mu_{\mathrm{nf}}}{\rho_{\mathrm{nf}}}+\left(\frac{\partial^{2} \mathrm{u}}{\partial \mathrm{r}^{2}}+\frac{1}{\mathrm{r}} \frac{\partial \mathrm{u}}{\partial \mathrm{r}}\right)-\frac{\sigma_{\mathrm{nf}}}{\rho_{\mathrm{nf}}} \mathrm{B}_{0}^{2} \mathrm{u} \\
& u \frac{\partial T}{\partial x}+v \frac{\partial T}{\partial r}=\frac{k_{n f}}{\left(\rho_{c p}\right)_{n f}}\left(\frac{\partial^{2} T}{\partial r^{2}}+\frac{1}{r} \frac{\partial T}{\partial r}\right)-\frac{1}{\left(\rho c_{n f}\right)_{n f}} \frac{\partial q_{r}}{\partial r}(3)
\end{aligned}
$$

The boundary conditions are

$$
\begin{aligned}
& u=U_{w}, \quad v=0 \quad k f \frac{\partial T}{\partial r}=-q_{w^{x}} \text { at } r=a \\
& \begin{array}{ll}
\frac{\partial T}{\partial r}=-h_{s}\left(T-T_{\infty}\right) & \text { as } r \rightarrow \infty
\end{array} \\
& u \rightarrow 0, \quad T \rightarrow T_{\infty} \quad
\end{aligned}
$$

The radiative heat flux $q_{r}$ is simplified by the Rosseland diffusion approximation [33] as

$q_{r}=\frac{-4 \sigma}{3\left(\alpha_{s}+\sigma_{s}\right)} \frac{\partial T^{4}}{\partial r}$

Where $\sigma$ is the Stefan-Boltzmann constant, $\alpha_{r}$ is the Rosseland mean absorption factors and $\sigma_{s}$ is the scattering factor. Let us consider that the fluid -phase temperature differences within the flow are sufficiently small as reported in the work [34]. So that $T^{4}$ may be expanded as a linear function of temperature $T$ and neglecting higher order terms as

$$
T^{4} \approx 4 T_{\infty}^{3} T-3 T_{\infty}^{4}
$$

$q_{r}=\frac{-16 \sigma T_{\infty}^{3}}{3\left(\alpha_{s}+\sigma_{s}\right)} \frac{\partial T}{\partial r}$

It is worth mentioning here that use of the Rosseland diffusion approximation is valid the interior of a medium but it is not employed near the boundaries. It is good only from optically thick boundary layer. Since expression (8) does not contain any term for the radiation from the boundary surface therefore is not valid to predict a complete description of this physical situation near the surface. In the other words the boundary surface effects are negligible in the interior of an optically thick boundary layer region. Which is due to the fact that the radiation from the boundaries becomes very weak before reaching the interior.

In the above $q_{r}$ is radiative heat flux, $h_{s}$ is the convective heat transfer coefficient, $r, x$ are axial and radial coordinates of the cylinder respectively, $u, v$ are the velocity components along $x$ and $r$ directions. $T$ is temperature, $\rho_{n f}, \mu_{n f}$ and $\alpha_{n f}$ are the density, dynamic viscosity, thermal diffusivity of the nanofluid respectively is given by

$$
\begin{aligned}
& \mu_{n f}=\frac{\mu_{f}}{(1-\phi)^{2.5}}, \rho_{n f}=(1-\phi) \rho_{f}+\phi \rho_{s} \\
& \left(\rho_{c_{p}}\right)_{n f}=(1-\phi)\left(\rho_{p}\right)_{f}+\phi\left(\rho_{c}\right)_{f}, v_{n f}=\frac{\mu_{n f}}{\rho_{n f}}
\end{aligned}
$$

$$
\frac{k_{n f}}{k_{f}}=\frac{\left(k_{s}+2 k_{f}\right)-2 \phi\left(k_{f}-k_{s}\right)}{\left(k_{s}+2 k_{f}\right)+\phi\left(k_{f}-k_{s}\right)}, \alpha_{n f}=\frac{k_{n f}}{\left(\rho_{c}\right)_{n f}}
$$

In the above equations, $\rho_{s}$ is the reference density of the solid fraction, $\rho_{f}$ is the reference density of the fluid fraction, $k_{s}$ is the thermal conductivity of the solid fraction, $c_{p}$ is the specific heat at constant pressure, $\mu_{f}$ is the viscosity of the fluid fraction, $\Phi$ is the solid volume fraction of a nano fluid, and $k_{n f}$ is the thermal conductivity of the nano fluid.

The following variables are defined

$$
\eta=\frac{\mathrm{r}^{2}-\mathrm{a}^{2}}{2 \mathrm{a}}\left(\frac{\mathrm{U}_{\mathrm{W}}}{v_{\mathrm{f}} \mathrm{x}}\right)^{1 / 2}, \psi=\left(v_{\mathrm{f}} \mathrm{U}_{\mathrm{W}} \mathrm{x}\right)^{1 / 2} \mathrm{af}(\eta), \theta=\frac{\mathrm{T}-\mathrm{T}_{\infty}}{\mathrm{T}_{\mathrm{f}}-\mathrm{T}_{\infty}}
$$

Here the stream function is $\psi \cdot$ which defined as $u=r^{-1} \frac{\partial \psi}{\partial r}$ and $v=-r^{-1} \frac{\partial \psi}{\partial x}$ this satisfies the equation of continuity.

$$
\begin{gathered}
(1+2 \gamma \eta) \mathrm{f}^{\prime \prime}+2 \gamma \mathrm{f}^{\prime \prime}+(1-\Phi)^{2.5}\left[1-\Phi+\Phi\left(\frac{\rho_{\mathrm{S}}}{\rho_{\mathrm{f}}}\right)\right]\left(\mathrm{ff}^{\prime \prime}-\mathrm{f}^{\prime 2}\right) \\
-(1-\Phi)^{2.5} \mathrm{Mf}^{\prime}=0
\end{gathered}
$$

$\left(\frac{\mathrm{knf}_{\mathrm{nf}}}{\mathrm{kf}_{\mathrm{f}}}\right) \frac{1}{\operatorname{Pr}}\left(1+\frac{4}{3} \mathrm{Rd}\right)\left[(1+2 \gamma \eta) \theta^{\prime \prime}+2 \gamma \theta^{\prime}\right]$

Now expression (5) reduces to the form 


$$
\begin{aligned}
& +\left[1-\Phi+\Phi \frac{\left(\rho_{\mathrm{c}}\right)_{\mathrm{s}}}{\left(\rho_{\mathrm{c}}\right)_{\mathrm{f}}}\right]\left(\mathrm{f} \theta^{\prime}-\mathrm{f}^{\prime} \theta\right)=0 \\
& f(0)=0 f^{\prime}(0)=1 \theta^{\prime}(0)=B_{i}(1-\theta(0))
\end{aligned}
$$

$f^{\prime} \rightarrow 0, \quad \theta \rightarrow 0$ as $\quad \eta \rightarrow \infty$

Here $\operatorname{Pr}$ is the Prandtl number, $M$ is the Magnetic parameter, $\gamma$ Curvature parameter, $R d$ is the radiation parameter and $B_{i}$ is local Boit number.

$\gamma=\left(\frac{l_{\nu_{\mathrm{f}}}}{\mathrm{U}_{0 \mathrm{a}}{ }^{2}}\right)^{1 / 2}, \operatorname{Pr}=\frac{\mu_{\mathrm{f}}\left(\rho_{\mathrm{c}_{\mathrm{p}}}\right)}{\rho_{\mathrm{f}} \mathrm{k}_{\mathrm{f}}}, \mathrm{M}=\frac{\sigma_{\mathrm{nf}} \mathrm{B}_{0}^{2}}{\rho_{\mathrm{f}} \mathrm{U}_{0}}$

$\beta=\delta\left(\frac{\mathrm{U}_{0}}{1 v_{\mathrm{f}}}\right), \mathrm{Rd}=\frac{-4 \sigma \mathrm{T}_{\infty}^{3}}{\mathrm{kf}_{\mathrm{f}}\left(\alpha_{\mathrm{r}}+\sigma_{\mathrm{S}}\right)}, \mathrm{B}_{\mathrm{i}}=\mathrm{h}_{\mathrm{s}} \frac{\mathrm{a}}{\mathrm{r}} \sqrt{\frac{v_{\mathrm{f}} \mathrm{X}}{\mathrm{U}_{\mathrm{W}}}}$

We know that $\gamma=0$ and $\gamma=1$ for plate and cylinder respectively. $C_{f}$ is called skin friction coefficient and $N u_{x}$ is local Nusselt number. These quantities can be written as

$$
C_{f}=\frac{\tau_{w}}{\rho_{f} U_{w}^{2}}, N u_{x}=\frac{x q_{w}}{k_{f}\left(T f-T_{\infty}\right)}
$$

Here $\mathrm{q}_{\mathrm{w}}$ is heat flux from the plate, $\tau_{\mathrm{w}}$ is the skin friction those values are

$$
\tau_{w}=\mu_{n f}\left(\frac{\partial u}{\partial r}\right)_{r=a}, q_{w}=-k f\left(\left(1+\frac{16 \sigma_{T}^{3}}{3\left(\alpha_{r}+\sigma_{s}\right.}\right) \frac{\partial T}{\partial r}\right)_{r=a}
$$

We have

$$
\begin{aligned}
& \operatorname{Re}_{x}{ }^{1 / 2} C_{f}=\frac{1}{(1-\Phi)^{2.5}} f^{\prime \prime}(0) \\
& \operatorname{Re}_{x}{ }^{-1 / 2} N u_{x}=\frac{-k n f}{k_{f}}\left(1+\frac{4}{3} R d\right) \theta^{\prime}(0)
\end{aligned}
$$

For a pure fluid $\Phi=\mathrm{O}$, In the absence of slip condition $\beta=\mathrm{O}$ and magnetic field $M=0$.

\section{Solution of the Problem}

Substitute the above values in equations (11) and (12), we have $(1+2 \gamma \eta) f^{\prime \prime \prime}+2 \gamma f^{\prime \prime}+(1-\Phi)^{2.5} A_{1}\left(f f^{\prime \prime}-f^{\prime 2}\right)-(1-\Phi)^{2.5} M f^{\prime}=0$

$A_{3} \frac{1}{\operatorname{Pr}}\left(1+\frac{4}{3} R d\right)\left[(1+2 \gamma \eta) \theta^{\prime \prime}+2 \gamma \theta^{\prime}\right]+A_{2}\left(f \theta^{\prime}-f^{\prime} \theta\right)=0$

Finite difference method:

$$
\begin{aligned}
& A_{1}=(1-\Phi)^{2.5}, \quad A_{2}=(1-\Phi)+\Phi \frac{\rho_{s}}{\rho_{f}} \\
& A_{3}=\frac{k_{n f}}{k_{f}}, \quad A_{4}=(1-\Phi)+\Phi \frac{\left(\rho c_{p}\right)_{s}}{\left(\rho c_{p}\right)_{f}}
\end{aligned}
$$

In order to solve the above equations (18)-(19), we are using new dependent variables $p(\eta), q(\eta), \theta(\eta)$, and $t(\eta)$ such that

$$
\begin{aligned}
& f^{\prime}=p, p^{\prime}=q, \theta^{\prime}=t \\
& {\left[(1+2 \gamma \eta) q^{\prime}+2 \gamma q\right]+A_{1} A_{2}\left[\frac{f q}{2}-p^{2}\right]-A_{1} M p=0}
\end{aligned}
$$

$$
\left(A_{3}+\frac{4}{3} N r\right)\left[(1+2 \gamma \eta) t^{\prime}+\gamma t\right]+\left(A_{3} \gamma t\right)+\frac{p_{r}}{2} \cdot A_{4} f t=0
$$

Now the boundary conditions () becomes

$$
\begin{aligned}
& p(0)=1, f(0)=0, \theta(0)=1 \\
& p(\eta) \rightarrow 0, \theta(\eta) \rightarrow 0, \text { as } \eta \rightarrow \infty
\end{aligned}
$$

Define

$\eta_{0}=0, \quad \eta_{j}=\eta_{j-1}+h_{j}, \quad \eta_{J}=\eta_{\infty}$

Where the segment $\eta_{j-1} \eta_{j}$ with midpoint $\eta_{j-\frac{1}{2}}, h_{j}$ as $\Delta \eta$ spacing and index $j=1,2 \ldots . \mathrm{J}$

The finite difference approximations for the equations (20) to (22) are

$$
f_{j}-f_{j-1}-\frac{1}{2} h_{j}\left(p_{j}+p_{j-1}\right)=0
$$

$p_{j}-p_{j-1}-\frac{1}{2} h_{j}\left(q_{j}+q_{j-1}\right)=0$

$\theta_{j}-\theta_{j-1}-\frac{1}{2} h_{j}\left(t_{j}+t_{j-1}\right)=0$

$$
(1+2 \gamma \eta)\left(\mathrm{q}_{\mathrm{j}}-\mathrm{q}_{\mathrm{j}-1}\right)+\mathrm{h}_{\mathrm{j}} \gamma\left(\mathrm{q}_{\mathrm{j}}+\mathrm{q}_{\mathrm{j}-1}\right)+\frac{\mathrm{A}_{1} \mathrm{~A}_{2}}{2} \mathrm{~h}_{\mathrm{j}}(\mathrm{fq})_{\mathrm{j}-\frac{1}{2}}
$$$$
-\mathrm{A}_{1} \mathrm{~A}_{2} \mathrm{~h}_{\mathrm{j}} \mathrm{p}_{\mathrm{j}-1}^{2}-\mathrm{A}_{1} \mathrm{Mp}_{\mathrm{j}-\frac{1}{2}}=0
$$

$$
\begin{aligned}
& \left(\mathrm{A}_{3}+\frac{4}{3} \mathrm{Nr}\right)\left[(1+2 \gamma \eta)\left(\mathrm{t}_{\mathrm{j}}-\mathrm{t}_{\mathrm{j}-1}\right)+\mathrm{h}_{\mathrm{j}} \gamma\left(\mathrm{t}_{\mathrm{j}}+\mathrm{t}_{\mathrm{j}-1}\right)\right] \\
& +\left(\mathrm{A}_{3} \mathrm{~h}_{\mathrm{j}} \gamma \mathrm{t}_{\mathrm{j}-\frac{1}{2}}\right)+\frac{\mathrm{p}_{\mathrm{r}}}{2} \cdot \mathrm{A}_{4} \mathrm{~h}_{\mathrm{j}}(\mathrm{ft})_{\mathrm{j}-\frac{1}{2}}=0
\end{aligned}
$$

Equations (24) - (28), $j=1,2, \ldots \ldots J$, the boundary layer thickness $\eta_{J}$ exceeds the edge of the boundary for extremely high values, the new boundary conditions are

$$
f_{0}=0, p_{0}=1, \theta_{0}=1, p_{J}=0, \theta_{J}=0
$$

Newton's method.

Linearising the non-linear system (24)-(28), by using Newton's method, introduce

$$
\begin{aligned}
& f_{j}^{(k+1)}=f_{j}^{(k)}+\delta f_{j}^{(k)}, \quad p_{j}^{(k+1)}=p_{j}^{(k)}+\delta p_{j}^{(k)}, \\
& q_{j}^{(k+1)}=q_{j}^{(k)}+\delta q_{j}^{(k)},
\end{aligned}
$$


$\theta_{j}^{(k+1)}=\theta_{j}^{(k)}+\delta \theta_{j}^{(k)}, t_{j}^{(k+1)}=t_{j}^{(k)}+\delta t_{j}^{(k)}$

Where $\mathrm{k}=0,1,2$...

Using the above equation (13) and dropping ' $\mathrm{k}$ ' (for simplicity) we get,

$$
\begin{aligned}
& \delta f_{1}-\delta f_{0}-\frac{h_{1}}{2} \delta p_{1}-\frac{h_{2}}{2} \delta p_{0}=\left(r_{1}\right)_{j-\frac{1}{2}} \\
& \delta p_{1}-\delta p_{0}-\frac{h_{1}}{2} \delta q_{1}-\frac{h_{2}}{2} \delta q_{0}=\left(r_{2}\right)_{j-\frac{1}{2}} \\
& \delta \theta_{1}-\delta \theta_{0}-\frac{h_{1}}{2} \delta t_{1}-\frac{h_{2}}{2} \delta t_{0}=\left(r_{3}\right)_{j-\frac{1}{2}} \\
& \left(\mathrm{a}_{1}\right) \delta \mathrm{q}_{\mathrm{j}}+\left(\mathrm{a}_{2}\right) \delta \mathrm{q}_{\mathrm{j}-1}+\left(\mathrm{a}_{3}\right) \delta \mathrm{f}_{\mathrm{j}}+\left(\mathrm{a}_{4}\right) \delta \mathrm{f}_{\mathrm{j}-1} \\
& +\left(\mathrm{a}_{5}\right) \delta \mathrm{p}_{\mathrm{j}}+\left(\mathrm{a}_{6}\right) \delta \mathrm{p}_{\mathrm{j}-1}=\left(\mathrm{r}_{4}\right)_{\mathrm{j}-\frac{1}{2}} \\
& \left(\mathrm{~b}_{1}\right) \delta \mathrm{t}_{\mathrm{j}}+\left(\mathrm{b}_{2}\right) \delta \mathrm{t}_{\mathrm{j}-1}+\left(\mathrm{b}_{3}\right) \delta \mathrm{f}_{\mathrm{j}} \\
& +\left(\mathrm{b}_{4}\right) \delta \mathrm{f}_{\mathrm{j}-1}=\left(\mathrm{r}_{5}\right)_{\mathrm{j}-\frac{1}{2}}
\end{aligned}
$$

Where

$$
\begin{aligned}
& \left(a_{1}\right)=(1+2 \gamma \eta)+h_{j} \gamma+\frac{1}{4} h_{j} A_{1} A_{2} f_{j-\frac{1}{2}} \\
& \left(a_{2}\right)=-(1+2 \gamma \eta)+h_{j} \gamma+\frac{1}{4} h_{j} A_{1} A_{2} f_{j-\frac{1}{2}} \\
& \left(a_{3}\right)=\frac{1}{4} h_{j} A_{1} A_{2} f_{j-\frac{1}{2}}
\end{aligned}
$$

$$
\begin{aligned}
& \left(a_{4}\right)=\left(a_{3}\right) \\
& \left(a_{5}\right)=-A_{1} A_{2} h_{j-\frac{1}{2}}-\frac{1}{2} A_{1} M h_{j} \\
& \left(a_{5}\right)=\left(a_{6}\right) \\
& \left(b_{1}\right)=\left(A_{3}+\frac{4}{3} N r\right)(1+2 \gamma \eta)+h_{j} \gamma\left(A_{3}+\frac{4}{3} N r\right)+\frac{A_{3} r h_{j}}{2}+\frac{p_{r} A_{4}}{4} h_{j} f_{j-\frac{1}{2}} \\
& \left(b_{2}\right)=-\left(A_{3}+\frac{4}{3} N r\right)(1+2 \gamma \eta)+h_{j} \gamma\left(A_{3}+\frac{4}{3} N r\right)+\frac{A_{3} r h_{j}}{2}+\frac{p_{r} A_{4}}{4} h_{j} f_{j-\frac{1}{2}}
\end{aligned}
$$

$$
\begin{aligned}
& \left(b_{3}\right)=f_{j-\frac{1}{2}} \\
& \left(b_{4}\right)=\left(b_{3}\right)
\end{aligned}
$$

Now the boundary conditions in (12) become,

$$
\delta f_{0}=0, \delta p_{0}=0, \delta \theta_{0}=0
$$

Block- Elimination Method:

Solving the linearised differential equations (24) to (28) by using block- elimination method as outlined by Cebeci and Bradshaw [35], as the system is in block tri-diagonal structure. Generally this structure contains constants or variables, whereas here it is noted that it contains block matrices. Writing equations (24) to (28) in matrix vector form,
$\mathrm{A} \delta=\mathrm{r}$

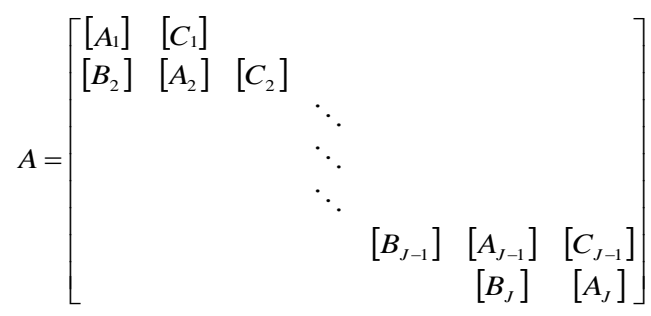

$\delta=\left[\begin{array}{c}{\left[\delta_{1}\right]} \\ {\left[\delta_{2}\right]} \\ \vdots \\ {\left[\delta_{J-1}\right]} \\ {\left[\delta_{J}\right]}\end{array}\right], \quad$ and $r=\left[\begin{array}{c}{\left[r_{1}\right]} \\ {\left[r_{2}\right]} \\ \vdots \\ {\left[r_{J-1}\right]} \\ {\left[r_{J}\right]}\end{array}\right]$

The entries of the matrices are,

$\left[A_{1}\right]=\left[\begin{array}{ccccc}0 & 0 & 1 & 0 & 0 \\ \frac{-h_{1}}{2} & 0 & 0 & \frac{-h_{1}}{2} & 0 \\ 0 & \frac{-h_{1}}{2} & 0 & 0 & \frac{-h_{1}}{2} \\ \left(a_{2}\right)_{1} & 0 & \left(a_{3}\right)_{1} & \left(a_{1}\right)_{1} & 0 \\ 0 & \left(b_{2}\right)_{1} & \left(b_{3}\right)_{1} & 0 & \left(b_{1}\right)_{1}\end{array}\right]$

$2 \leq \mathrm{j} \leq \mathrm{J}$

$\left[A_{j}\right]=\left[\begin{array}{ccccc}\frac{-h_{2}}{2} & 0 & 1 & 0 & 0 \\ -1 & 0 & 0 & \frac{-h_{2}}{2} & 0 \\ 0 & -1 & 0 & 0 & \frac{-h_{2}}{2} \\ \left(a_{6}\right)_{j} & 0 & \left(a_{3}\right)_{j} & \left(a_{1}\right)_{j} & 0 \\ 0 & 0 & \left(b_{3}\right)_{j} & 0 & \left(b_{1}\right)_{j}\end{array}\right]$

$\left[B_{\mathrm{j}}\right]=\left[\begin{array}{ccccc}0 & 0 & -1 & 0 & 0 \\ 0 & 0 & 0 & \frac{-h_{2}}{2} & 0 \\ 0 & 0 & 0 & 0 & \frac{-h_{2}}{2} \\ 0 & 0 & \left(a_{4}\right)_{j} & \left(a_{2}\right)_{j} & 0 \\ 0 & 0 & \left(b_{4}\right)_{j} & 0 & \left(b_{2}\right)_{j}\end{array}\right]$

$1 \leq \mathrm{j} \leq \mathrm{J}-1$

$\left[C_{\mathrm{j}}\right]=\left[\begin{array}{ccccc}\frac{-h_{1}}{2} & 0 & 0 & 0 & 0 \\ 1 & 0 & 0 & 0 & 0 \\ 0 & 1 & 0 & 0 & 0 \\ \left(a_{5}\right)_{j} & 0 & 0 & 0 & 0 \\ 0 & 0 & 0 & 0 & 0\end{array}\right]$

$2 \leq \mathrm{j} \leq \mathrm{J}$ 
$\left[\delta_{1}\right]=\left[\begin{array}{c}\delta q_{0} \\ \delta t_{0} \\ \delta f_{1} \\ \delta q_{1} \\ \delta t_{1}\end{array}\right], \quad\left[\delta_{J}\right]=\left[\begin{array}{c}\delta q_{j-1} \\ \delta t_{j-1} \\ \delta f_{j} \\ \delta q_{j} \\ \delta t_{j}\end{array}\right]$,

And $1 \leq \mathrm{j} \leq \mathrm{J}$

$\left[r_{1}\right]=\left[\begin{array}{l}\left(r_{1}\right)_{j-1 / 2} \\ \left(r_{2}\right)_{j-1 / 2} \\ \left(r_{3}\right)_{j-1 / 2} \\ \left(r_{4}\right)_{j-1 / 2} \\ \left(r_{5}\right)_{j-1 / 2}\end{array}\right]$

Suppose the matrix A in equation (39), is non-singular and solving the equation using factorization method.

Write

$\mathrm{A}=\mathbf{L U}$,

Where

$\mathrm{L}=\left[\begin{array}{ccccc}{\left[\alpha_{1}\right]} & & & & \\ {\left[\beta_{1}\right]} & {\left[\alpha_{2}\right]} & {\left[C_{2}\right]} & & \\ & & & \ddots & \\ & & \ddots & \\ & & & {\left[\alpha_{J-1}\right]} & \\ & & & {\left[\beta_{J}\right]} & {\left[\alpha_{J}\right]}\end{array}\right]$

and<smiles>CC(C)C1CCCC1</smiles>

Here matrix I is $5 \times 5$ identity matrix, $\left[\alpha_{i}\right]$ and $\left[\Gamma_{i}\right]$ are 5 X 5 square matrices in which entries are given by the below equations:

$\left[\alpha_{1}\right]=\left[A_{1}\right]$

$\left[A_{1}\right]\left[\Gamma_{1}\right]=\left[C_{1}\right]$,

$\left[\alpha_{i}\right]=\left[A_{1}\right]-\left\lfloor B_{j}\right\rfloor\left\lfloor\Gamma_{j-1}\right\rfloor, \mathrm{j}=2,3 \ldots \ldots . . . \mathrm{J}$

$\left[\alpha_{i}\right]\left\lfloor\Gamma_{j}\right\rfloor=\left\lfloor C_{j}\right\rfloor, \mathrm{j}=2,3 \ldots . . . \mathrm{J}-1$

Using these equations we get,

$\mathrm{LU} \delta=\mathrm{r}$,

Define

$\mathrm{U} \delta=\omega$,

Now (50) becomes

$\mathrm{L} \omega=\mathrm{r}$,
Here

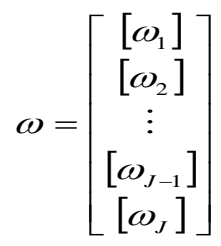

Here the entries of $\omega$ are determined by the below equations.

$\left[\alpha_{1}\right]\left[\omega_{1}\right]=\left[r_{1}\right]$

$\left[\alpha_{i}\right\rfloor\left\lfloor\omega_{j}\right\rfloor=\left\lfloor r_{j}\right\rfloor-\left\lfloor B_{j}\right\rfloor\left\lfloor\omega_{j-1}\right\rfloor, 2 \leq \mathbf{j} \leq \mathbf{J}$

After finding the elements of $\omega$ and using the following relations we will get the solution for $\delta$.

$\left[\delta_{1}\right]=\left[\omega_{1}\right]$

$\left[\delta_{i}\right]=\left\lfloor\omega_{j}\right\rfloor-\left\lfloor\Gamma_{j}\right\rfloor\left\lfloor\delta_{j+1}\right\rfloor, 1 \leq \mathbf{j} \leq \mathbf{J}-1$

Repeat the calculations until we get the convergence criterion, i.e.

$\left|\delta v_{0}^{i}\right| \leq \varepsilon_{1}$

Here $\varepsilon_{1}$ is the small chosen value.

\section{Results and Discussion}

Applied Keller box method to resolve two non-linear equations 11 and 12. The methods of computations made by using the symbolic computational software Matlab. We compared our outcome results with those of Akbar et al. [36], Sala Huddin et al. [37] and Malik et al. [38]. Our results agreed with them up to nine decimal places. These are noted in table 2.

The change in velocity and temperature for dissimilar values of magnetic nano particle volume fractions are tested for both flat plate and cylinder and these are shown in Fig.s 2 and 3. We observed that velocity as well as temperature also increases with increasing accordingly with solid volume fraction. This increase is comparatively high for cylinder than the flat plate. When $\Phi=0$, $0.1,0.2$, with $M=0.5, P r=7$ (water), $R d=0.5$ and $B i=0.1$. This emphasizes the fact that, the volume fraction of nano particle is directly proportional to both thermal conductivity as well as thermal boundary layer thickness.

Also it is tested for changeable values of magnetic parameter how both the velocity temperature profiles changes. We observed that on increasing the values of magnetic parameter the velocity profiles are inversely proportional to temperature profiles. The physics behind these results is that the transverse magnetic field which is perpendicular to the direction of electrically conducting fluid leads to a dragging force which is known as Lorentz force which opposes or contradicts to the smooth motion of fluid this causes a retardation effect, means decrease in velocity and increase in the thickness of boundary layer. It is shown in Fig.s 4 and 5 .

Fig. (6) explains the impact of thermal radiation on temperature profile of magnetic nano particle and the remaining parameters fixed at $\Phi=0.1, \operatorname{Pr}=7, M=0.5, B i=0.1$ both for flat plate and cylinder. It is marked that the temperature profile of the magnetic nano particle is an ascending function of thermal radiation parameter this leads to the enhancement of thickness of thermal boundary layer. The reason behind this is the increase in the thermal radiation, leads to decrease in Rosseland radiation absorptive. So the deviation of radiative heat flux $\mathrm{q}_{\mathrm{r}}$ increases with decrease in the absorption coefficient consequently the rate of radiative heat transferred to the fluid increases then both temperature as well as thickness of boundary layer increases.

Fig. (7) tells about the effect of Biot number (convective heating) over temperature profile of magnetic nano particle when $\mathrm{Bi}=0$ (no convective heating), $0.1,0.2$ and 0.3 with $\Phi=0.1, \operatorname{Pr}=7, M$ $=0.5, R d=0.5$ for both flat plate and cylinder. We noticed that 
temperature is an increasing function of parameter $\boldsymbol{B}_{i}$ we have the stronger convection results for high surface temperatures which leads to go deeper into the stagnant fluid. From Fig. (8), we noticed that magnitude of skin friction coefficient is directly proportional to the values of both the magnetic field parameter as well as magnetic nano particle volume fraction. Also remarked that the coefficient of drag skin friction is more for cylinder than that of flat plate. Here the negative value of coefficient of skin friction means the stretching cylinder employ a dragging force over the surface of the fluid and the positive values employ the opposite.

Fig. (9) shows that drag Nusselt number (rate of heat transfer) is inversely proportional to the magnetic field parameter, however it is directly proportional to the magnetic nano particle volume fraction. It's true, since the thermal conductivity increases with nano particle volume fraction. This implies the acceleration of heat transfers rate entire the boundary layer. Increase in both thermal radiation parameter and the convective heating parameter shows increase in drag Nusselt number.

In table 3, we compared the results across magnetic and non magnetic nano particles. Here we have chosen $\mathrm{Al}_{2} \mathrm{O}_{3}$ as non magnetic nano particle. Thermal conductivity of $\mathrm{Al}_{2} \mathrm{O}_{3}$ is $40 \mathrm{~W} / \mathrm{m}-\mathrm{k}$. Because $\mathrm{Al}_{2} \mathrm{O}_{3}$ is having high thermal conductivity, than magnetic nano particles, and Nusselt numbers of $\mathrm{Al}_{2} \mathrm{O}_{3}$ are high even in the absence of magnetic field. When magnetic field strength is increased all the magnetic nano particles arranged into same direction and comparatively show higher heat transfer rates, like non magnetic nano particle $\mathrm{Al}_{2} \mathrm{O}_{3}$. This collation shown in table 3 .

The heat transfer rate is high for $\mathrm{Al}_{2} \mathrm{O}_{3}$ when compared with that of magnetic nano particles when there is no magnetic field. Almost a percent difference between magnetic and non magnetic nano particles increase by increasing solid volume fraction of nano particles. When magnetic field strength is increased the magnetic nano particles join themselves, also the percent difference with $\mathrm{Al}_{2} \mathrm{O}_{3}$ decreases.

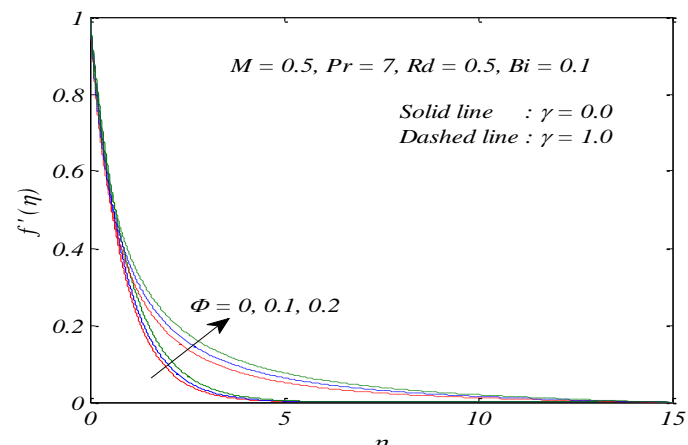

Fig. 2: Velocity profiles $f^{\prime}(\eta)$ under different $\Phi$

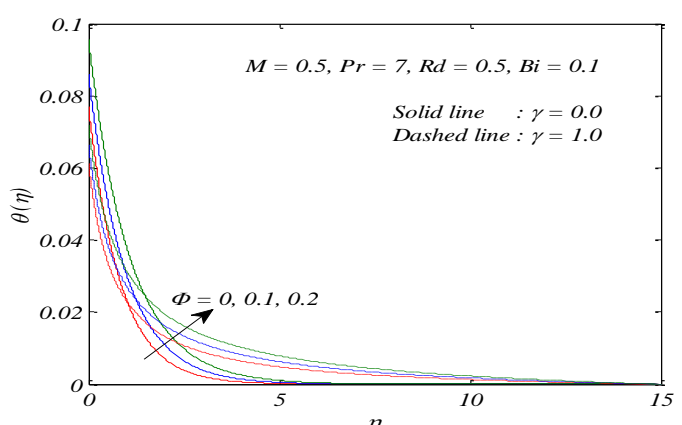

Fig. 3: Temperature profiles $\theta(\eta)$ under different $\Phi$

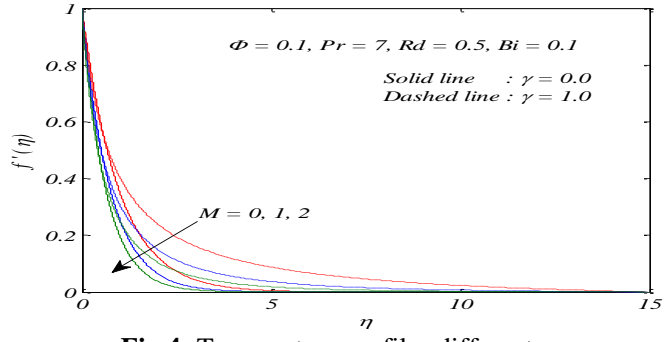

Fig.4: Temperature profiles different

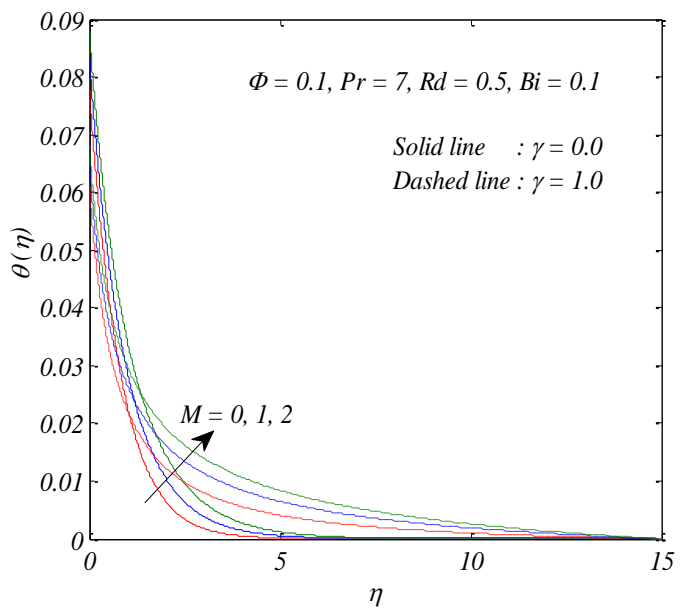

Fig. 5: Temperature profiles $\theta(\eta)$ under different $\boldsymbol{M}$

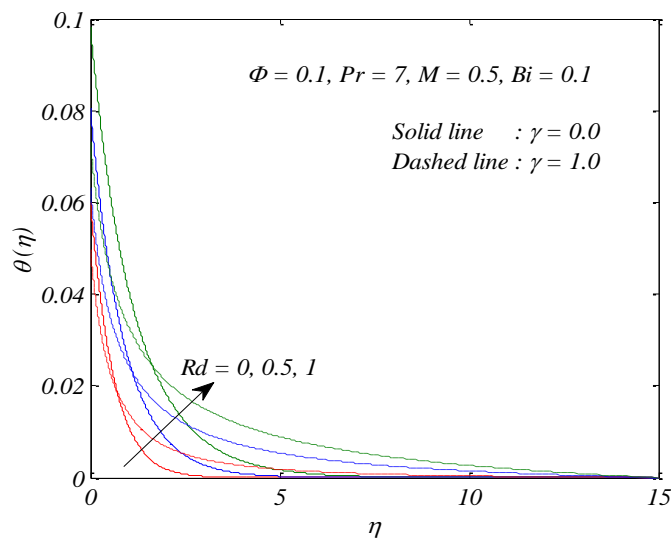

Fig. 6: Temperature profiles $\theta(\eta)$ under different $R d$

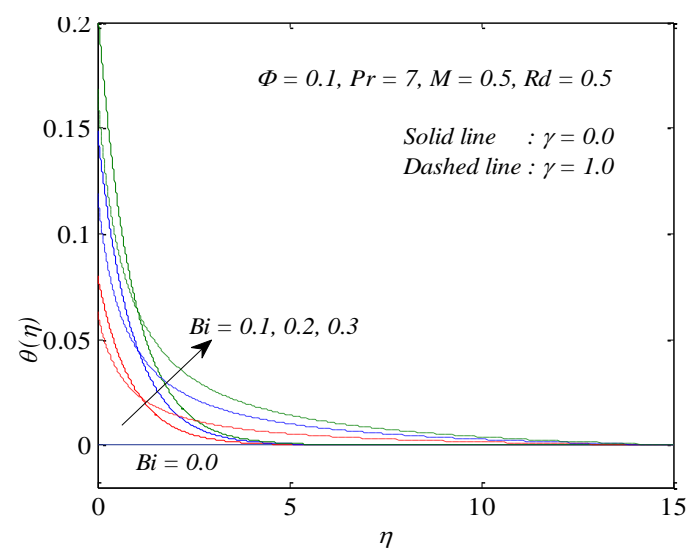

Fig. 7: Temperature profiles $\boldsymbol{\theta}(\boldsymbol{\eta})$ under different $\boldsymbol{B i}$ 


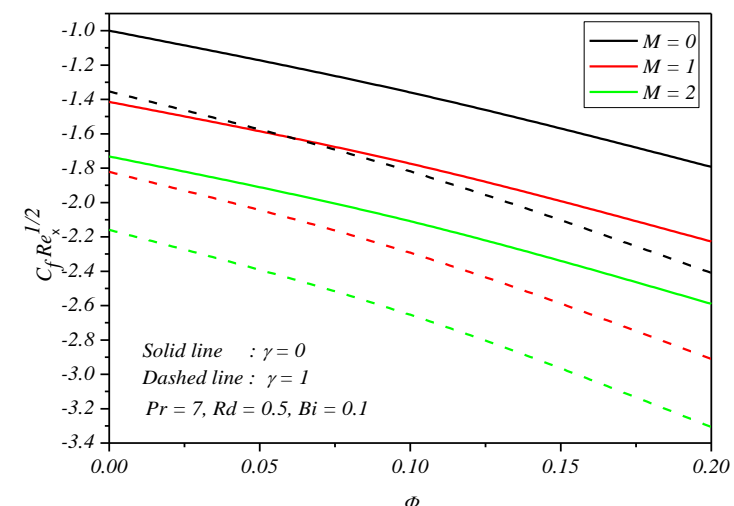

Fig. 8: $C_{f} \operatorname{Re}_{x}^{1 / 2}$ under different values of $M \& \Phi$

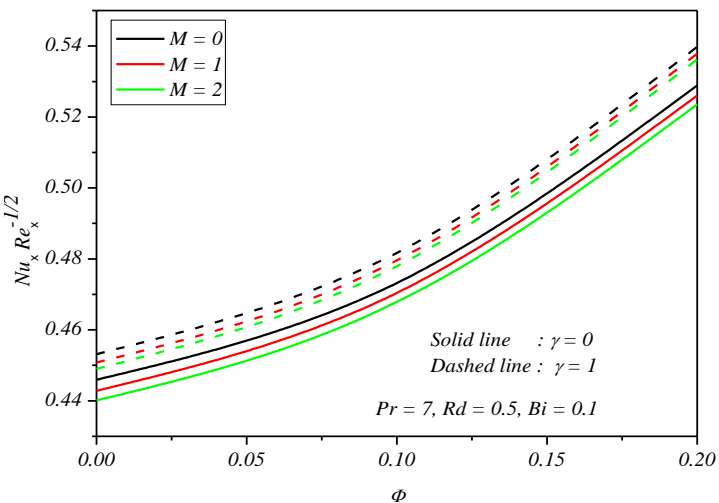

Fig. 9: $N u_{x} \operatorname{Re}_{x}^{-1 / 2}$ under different values of $M \& \Phi$

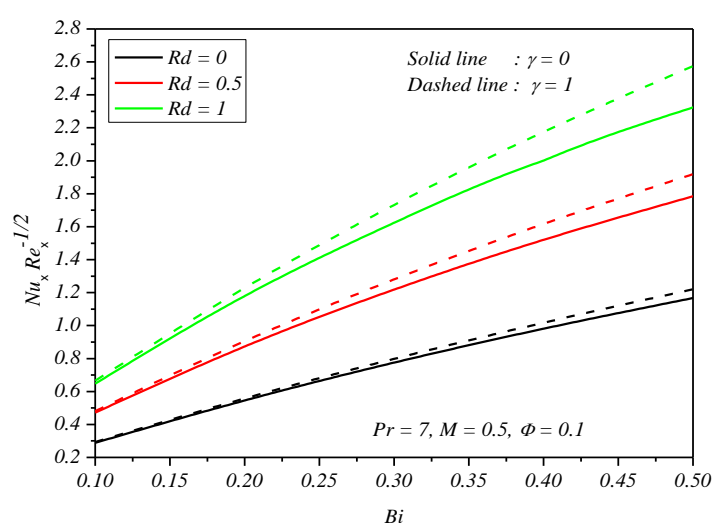

Fig. 10: $N u_{x} \operatorname{Re}_{x}^{-1 / 2}$ under different values of $R d \& B i$

Table1: Thermalphysical properties of base fluid/water and nanoparticle/magnetite $[19,20]$.

\begin{tabular}{|c|c|c|c|}
\hline Physical properties & $\begin{array}{c}\text { Water/base } \\
\text { fluid }\end{array}$ & Magnetite/Magnetic & $\begin{array}{c}\text { Al2O3/Non- } \\
\text { magnetite }\end{array}$ \\
\hline$\rho\left(k g / m^{3}\right)$ & 997 & 5180 & 3970 \\
\hline$c_{p}(J / k g K)$ & 4179 & 670 & 765 \\
\hline$k(W / m K)$ & 0.613 & 9.7 & 40 \\
\hline
\end{tabular}

Table 2: Comparison of $f^{\prime \prime}(0)$ for different values of $M$ in the absence of $\Phi$ for clear fluids.

\begin{tabular}{|c|c|c|c|c|}
\hline$M$ & Akbar et al. [36] & $\begin{array}{c}\text { Salahuddin } \\
\text { et al. [37] }\end{array}$ & $\begin{array}{c}\text { Malik et al. } \\
{[38]}\end{array}$ & $\begin{array}{c}\text { Present out } \\
\text { put }\end{array}$ \\
\hline 0 & 1 & 1 & 1 & 1 \\
0.5 & -1.11803 & -1.11801 & -1.118105 & -1.11803399 \\
1 & -1.41421 & -1.41418 & -1.14415 & -1.41421356 \\
5 & -2.44949 & -2.44942 & -2.44947 & -2.44948974 \\
10 & -3.31663 & -3.31656 & -3.31696 & -3.31662479 \\
100 & -10.04988 & -10.04981 & -10.04983 & -10.04987562 \\
500 & -22.38303 & -22.038293 & -22.38284 & -22.38302929 \\
1000 & -31.63839 & -31.63846 & -31.63851 & -31.63858404 \\
\hline
\end{tabular}

Table 3: Deviation of Nusselt numbers with magnetic field parameter and solid volume friction of magnetic and non - magnetic nanoparticles with $\operatorname{Pr}=7, B i=0.1, \quad \gamma=1$.

\begin{tabular}{|c|c|c|c|c|}
\hline M & $\Phi$ & 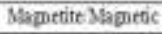 & Al: 0, Non-magerix & 5 \\
\hline \multicolumn{5}{|c|}{ Difference with $\mathrm{Al}_{2} \mathrm{O}_{3}$} \\
\hline \multirow[t]{4}{*}{0} & 0 & 0.45315832 & 0.45573460 & 0.027003362 \\
\hline & 0.05 & 0.45471045 & 0.48224043 & 0.03138648 \\
\hline & 0.1 & 0.48169334 & 0.50627906 & 0.04856159 \\
\hline & 0.2 & 0.53534119 & 0.59063948 & 0.05600555 \\
\hline \multirow[t]{4}{*}{1} & 0 & 0.45078048 & 0.45324207 & 0.02690082 \\
\hline & 0.05 & 0.45250107 & 0.47934974 & 0.03615638 \\
\hline & 0.1 & 0.47960258 & 0.50393838 & 0.04329122 \\
\hline & 0.2 & 0.53734817 & 0.58321499 & 0.03562555 \\
\hline \multirow[t]{4}{*}{2} & 0 & 0.4492220 & 0.45140788 & 0.02634323 \\
\hline & 0.05 & 0.46052835 & 0.47805819 & 0.02684323 \\
\hline & 0.1 & 0.47798724 & 0.50215632 & 0.04513059 \\
\hline & 0.2 & 0.53625599 & 0.55832160 & 0.05538933 \\
\hline
\end{tabular}

\section{Conclusions}

The present study investigates the magneto hydrodynamic flow and heat transfer analysis of ferrofluid along a stretching cylinder with thermal radiation and convective heating. The main findings of the study are:

By considering the thermal radiation and convective heating on energy equation we tested the magnetic hydro dynamic flow of ferrofluid along a flat plate and stretched cylinder.

The profiles of temperature, velocity, surface shear stress and Nusselt number are good in the case of stretching cylinder.

On increasing the magnetic field parameter, both the velocity, heat transfer rate at the surface decreases.

There is a decrease in percent difference in heat transfer rate of magnetic nano particles along with $\mathrm{Al}_{2} \mathrm{O}_{3}$ when magnetic field strength is increased.

The heat transfer increases due to thermal radiation and convective heating.

Current results have good agreement with the previous results up to nine decimals.

\section{References}

[1] Das SK, Choi SUS, Yu W \& Pradeep T (2007), Nanofluids-Science and Technology, John Wiley \& Sons Publishers, Hoboken, 2007.

[2] Buongiorno J (2006), Convective transport in nanofluids, ASME J. Heat Transfer 128, 240-250.

[3] Tiwari RK \& Das MK (2007), Heat transfer augmentation in a twosided lid-driven differentially heated square cavity utilizing nanofluids, International Journal of Heat Mass Tran fer 50 (2007) 2002-2018.

[4] Hady FM, Ibrahim FS \& Abdel-Gaied, SM (2012), Radiation effect on viscous flow of a nanofluid and heat transfer over a nonlinearly stretching sheet, Nanoscale. Res. Lett. 7, 229-236.

[5] Chamkha AJ, Abbasbandy S, Rashad AM \& Vajravelu K (2012), Radiation effects on mixed convection over a wedge embedded in a porous medium filled with a nanofluid, Transp. Porous Medium 91, 261-279.

[6] Chamkha AJ, Abbasbandy S, Rashad AM \& Vajravelu K (2013), Radiation effects on mixed convection about a cone embedded in a porous medium filled with a nanofluid, Meccanica 48, 275-28 .

[7] Bég OA \& Ferdows M (2013), Explicit numerical simulation of magnetohydrodynamic nanofluid flow from an exponential stretching sheet in porous media, Appl. Nanosci 1-15.

[8] EL-Kabeir SMM, Chamkha AJ \& Rashad AM (2014), The effect of thermal radiation on non-Darcy free convection from a vertical cylinder embedded in a nanofluid porous media, J. Porous Media 17 (3), 269-278 .

[9] Zhu J, Zheng L \& Zhang X (2015), Second-order slip MHD flow and heat transfer of nanofluids with thermal radiation and chemical reaction, Appl. Math. Mech. -Engl. Ed. 36 (9), 1131-1146.

[10] EL-Kabeir SMM, Modather M \& Rashad AM (2015), Effect of thermal radiation on mixed convection flow of a nanofluid about a solid sphere in a saturated porous medium under convective boundary condition, J. Porous Media 18(6) 569-584 . 
[11] Turkyilmazoglu M (2015), Analytical solutions of single and multiphase models for the condensation of nanofluid film flow and heat transfer, Eur. J. Mech. B. Flu- ids 53, 272-277.

[12] Berkovsky BM, Medvdev VF \& Krakov MS (1973), Magnetic Fluids, Engineering Ap- plications, Oxford University Press, Oxford .

[13] Rosensweig RE (1985), Ferrohydrodynamics, Cambridge University Press, London .

[14] Kandelousi MS \& Ellahi R (2015), Simulation of ferrofluid flow for magnetic drug tar- geting, Z. Naturforsch 1-10 .

[15] Zeeshan A, Ellahi R \& Hassan M (2014), Magnetohydrodynamic flow of water/ethylene glycol based nanofluids with natural convection through a porous medium, Eur. Phys. J. Plus 129 (2014) 261

[16] Malvandi A, Ghasemi A \& Ganji DD (2016), Thermal performance analysis of hydro- magnetic $\mathrm{Al} 2 \mathrm{O} 3$-water nanofluid flows inside a concentric microannulus con- sidering nanoparticle migration and asymmetric heating, Int. J. Therm. Sci. 109, 10-22 .

[17] Heysiattalab S, Malvandi A \& Ganji DD (2016), Anisotropic behavior of magnetic nanofluids (MNFs) at filmwise condensation over a vertical plate in presence of a uniform variable directional magnetic field, J. Mol. Liq. 219, 875-882 .

[18] Malvandi A, Heysiattalab S \& Ganji DD (2016), Effects of magnetic field strength and direction on anisotropic thermal conductivity of ferrofluids (magnetic nanoflu-ids) at filmwise condensation over a vertical cylinder, Adv. Powder Technol. 27 (4), 1539-1546

[19] Rashad AM (2017), Impact of thermal radiation on MHD slip flow of a ferrofluid over a non-isothermal wedge, J. Magn. Magn. Mater. 422, 25-31.

[20] Rashad AM (2017), Impact of anisotropic slip on transient three dimensional MHD flow of ferrofluid over an inclined radiate stretching surface, Journal of the Egyptian Mathematical Society 25, 230-237

[21] Hossain MA \& Takhar HS (1996), Radiation effects on mixed convection along a vertical plate with uniform surface temperature, Int. J. Heat Mass Transfer 31, 243-248.

[22] Hossain MA, Alim MA \& Rees D (1999), The effect of radiation on free convection from a porous vertical plate, Int. J. Heat Mass Transfer 42, 181-191.

[23] Raptis A, Perdikis C \& Takhar HS (2004), Effect of thermal radiation on MHD flow, Appl. Math. Comp., 153, 645-649.

[24] Sajid M \& Hayat T (2008), Influence of thermal radiation on the boundary layer flow due to an exponentially stretching sheet, Int. Commun. Heat and Mass Transfer 35 347-356.

[25] Hayat T, Abbas M, Sajid M \& Asghar S (2007), The influence of thermal radiation on MHD flow of a secondgrade fluid, Int. J. Heat Mass Transf50, 931-941.

[26] Molla MM, Saha SC, Khan MAI \& Hossain MA (2011), Radiation effects on natural convection laminar flow from a horizontal circular cylinder, Desalination and Water Treatment 30, 89-97.

[27] Ahmad H, Javed T \& Ghaffari A (2017), The influence of heat radiation on mixed convection boundary layer flow of a viscoelastic fluid over a circular cylinder with constant surface temperature, Thermophysics and Aeromechanics 24, 115-124.

[28] Aziz AA (2009), similarity solution for laminar thermal boundary layer over a flat plate with a convective surface boundary condition, Communications in Nonlinear Science and Numerical Simulation 14, 1064-1068.

[29] Makinde O D \& Aziz A (2011), Boundary layer flow of a nanofluid past a stretching sheet with a convective boundary condition, International Journal of Thermal Sciences 50, 1326-1332.

[30] Shehzad S A, Hayat T \& Alsaedi A (2012), Three-dimensional flow of Jeffery fluid with convective surface boundary conditions. International Journal of Heat and Mass Transfer 55, 3971-3976.

[31] Hayat T, Bilal Ashraf M, Alsaedi A \& Shehzad A (2015), Convective heat and mass transfer effects in three-dimensional flow of Maxwell fluid over a stretching surface with heat source, J. Cent. South Univ 22, 717-726.

[32] Zaib A, Bhattacharyya K, Khalid M \& Shafie S (2017), Thermal radiation effect on a mixed convection flow and heat transfer of the williamson fluid past an exponentially shrinking permeable sheet with a convective boundary condition, Journal of Applied Mechanics and Technical Physics 58 (3), 419-424.

[33] Rosseland S (1936), Theoretical Astrophysics, Oxford University Press, London.

[34] Raptis A, Perdikis C \& Takhar HS (2004), Effect of thermal radiation on MHD flow, Appl. Math. Comp., 153, 645-649.

[35] Cebeci T \& Bradshaw P (1984), Physical and computational aspects of convective heat transfer, first ed. Springer Verlag, New York
[36] Akbar NS, Ebaud A \& Khan ZH (2015), Numerical analysis of magnetic field effects on a Eyring-Powell fluid flow towards a stretching sheet, J. Magn. Magn. Mater. 382, 355-358.

[37] Salahuddin T, Malik MY, Hussain A, Bilal S \& Awais M (2016), MHD flow of Cattaneo-Christov heat flux model for Williamson fluid over a stretching sheet with variable thickness: using numerical approach, J. Magn. Magn. Mater. 401, 991-997.

[38] Malik MY, Khan M, Salahuddin T \& Khan I (2016) Variable viscosity and MHD flow in Casson fluid with Cattaneo-Christov heat flux model: Using Keller box method, Engineering Science and Technology, an International Journal 19, 1985-1992. 